\title{
Las Vinculaciones Establecidas por las Empresas Industriales Uruguayas y su Influencia sobre Sus Actividades de Innovación: el Caso de la Universidad y de Otros Agentes
}

\author{
Luis Silveira ${ }^{1^{*}}$ Agustín García ${ }^{1}$, Paula González ${ }^{1}$
}

\begin{abstract}
Resumen: En la actual economía globalizada a la innovación se la considera como un elemento clave de la competitividad de las firmas. La literatura económica ha puesto de manifiesto que la productividad está fuertemente influenciada por la capacidad de innovación que tienen las empresas u organizaciones de una región o nación.

Pero la innovación es impredecible tanto en términos de procesos como de resultados, lo que hace muy difícil definir y seleccionar políticas activas de incentivo. Es por eso que, tratar de comprender cómo innovan las empresas en una realidad concreta puede ayudar a mejorar no solo la comprensión del fenómeno, sino que también puede contribuir a generar conocimientos útiles para poder diseñar políticas de incentivos más adecuadas y eficientes.
\end{abstract}

En el presente paper se estudian las relaciones que las empresas establecen con los diferentes agentes del Sistema Nacional de Innovación (S.N.I.) y cómo éstas afectan las actividades de innovación que realizan. El fundamento de este enfoque se basa en la idea de que la innovación es un proceso abierto e interactivo y que parece estar circunscrito a determinadas situaciones. En este sentido, las políticas de innovación han reconocido que las relaciones que se establecen con las universidades y los centros tecnológicos y/o de investigación son claves para el fomento de la innovación industrial.

Palabras claves: Innovación; Sistemas de Innovación; Vinculación Empresa-Universidad.

Abstract: Today's in the global economy, innovation is seen as a key element in the competitiveness of firms. The economic literature has shown that productivity is strongly influenced by the innovation capability of companies and organizations in a region or nation.

But innovation is unpredictable, both in terms of processes and outcomes which makes it very difficult to define and select ac tive incentive policies. That's why, trying to understand how companies innovate in a concrete reality not only will help improve the understanding of the phenomenon, but also will help to generate knowledge to design appropriate and more efficient policies and incentives.

In this paper we study the relationships that companies establish with the different agents of the National Innovation System (NIS) and how they affect their innovation activities. The rationale for this approach is based on the idea that learning and innovation are open and interactive processes that appear to be confined to certain situations. In this sense, innovation policies have recognized that the relationships established with universities, technological and / or research centers are fundamental to the promotion of industrial innovation.

Keywords: Innovation; Innovation System; Business enterprise; University relationships.

$21^{\text {st }}$ International Association of Jesuit Business Schools (IAJBS)

$18^{\text {th }}$ Colleagues in Jesuit Business Education (CJBE)

\section{Introducción}

La literatura económica ha considerado que el bienestar de una sociedad está directamente relacionado con la capacidad de la economía de mantener altos niveles de empleo, de salarios, de rentas de capital. En la actualidad, en donde la alta competencia a nivel internacional ha supuesto una mayor presión sobre el medio ambiente, la disponibilidad de los recursos naturales, los valores y la cultura regionales o locales, los investigadores han agregado a la temática, la sostenibilidad ambiental, la salud, la felicidad de las personas, la problemática de la cohesión social, la calidad de vida, etc. En consecuencia, existe la convicción entre los investigadores de que los temas relevantes a estudiar ya no son solo los que están referidos a la dimensión económi$\mathrm{ca}$, sino que se deben de incluir temas que tienen que ver con aspectos sociales, culturales y medio ambientales (Aranguren \& Wilson, 2010).

El concepto de competitividad está estrechamente vinculado con la capacidad de un país o región de mantener y mejorar el bienestar de sus ciudadanos. Estos nuevos temas a los que hace referencia el párrafo anterior también deberían de ser tenidos en cuenta al estudiar este concepto. Por ejemplo, Wilson (2010) expresa que "la competitividad debe de ser definida en términos más amplios, como ser la capacidad que tiene un país de mejorar su índice de bienestar subjetivo, relativo a otros países." (Wilson, 2010, p. 255).

(1) Instituto de Competitividad, Universidad Católica del Uruguay

*Corresponding author: luis.silveira@ucu.edu.uy 
El profesor Michael Porter también ha incorporado parte de la nueva temática a su análisis de la competitividad. Un ejemplo es su más reciente definición de competitividad. Según dicho autor "un territorio es competitivo en la medida que sus empresas sean capaces de competir con éxito en la economía global y a la vez ofrecer altos y crecientes niveles de salarios y estándares de vida para el ciudadano medio" (Porter, 2012).

En particular, las actividades de innovación han sido señaladas como un elemento crítico para la mejora de la competitividad y el desarrollo económico. De acuerdo a Asheim et al. (2011), existe un fuerte consenso en que la innovación es el factor clave en la promoción de la competitividad en una economía globalizada y basada en el conocimiento. Así, a pesar de ser relativamente recientes, políticas regionales de incentivo a la innovación han tomado gran preponderancia en Europa y en otras partes del mundo (Visser \& Atzema, 2008). En palabras de Porter (1990): "la competitividad de una nación depende de la capacidad de su industria para innovar y mejorar. La empresa consigue ventaja competitiva mediante innovaciones".

En concreto, la innovación es vital para la competitividad de las empresas y para la creación y mantenimiento de empleos de calidad. Desde el punto de vista empresarial, la competitividad implica alcanzar y mantener, de forma sostenida, la capacidad para competir en el mercado (Camacho et al., 2010). La relación entre innovación, productividad y competitividad se ha vuelto un tema relevante a nivel académico y de política pública. Se ha insistido en que la productividad está fuertemente influenciada por la capacidad de innovación e interrelaciones que tienen las empresas e instituciones $u$ organizaciones de una región o nación (Asheim et al., 2011). Además, la innovación al formar parte de retroalimentaciones positivas a nivel de la firma, se articula con otras inversiones en acumulación de capacidades que contribuyen a mejorar su posicionamiento competitivo. Ello lleva a que exista la percepción de que la innovación es una actividad clave para el éxito de las firmas (Martínez-Sánchez et al., 2007).

$\mathrm{Al}$ ser la innovación un proceso abierto e interactivo, las políticas de innovación han reconocido que las relaciones que se establecen con las universidades, con centros tecnológicos y/o de investigación y otros agentes son claves para el fomento de la misma.

\section{Importancia del tema de estudio}

La innovación es un proceso complejo a través del cual las firmas transforman conocimientos en valor agregado. Existen muchas definiciones de innovación. Creemos que una de las que más se adecúa a la presente investigación es la definición desarrollada por Simmie (2006). Para este autor la innovación es la introducción nueva o con algún cambio de un producto, proceso, o servicio o una nueva forma de organizarse orientada al mercado.

El proceso de innovación debe de ser visto como una actividad medular de la empresa y como tal puede ser desarrollado y mejorado (Camacho et al., 2010). La idea clave es que la innovación ya no es un proceso que implica el involucramiento de un grupo reducido de personas (científicos e investigadores dedicados a la $\mathrm{I}+\mathrm{D}$ ), sino que es un proceso que debe integrar el aporte de diversos actores, en el cual además tiene fundamental importancia las interacciones y el ambiente externo en que está inmerso la empresa (Aranguren \& Wilson, 2010). El fundamento de este enfoque se basa en la idea de que el aprendizaje y la innovación son procesos interactivos (Lundvall, 1992) y que parecen estar circunscritos a determinadas regiones y/o situaciones (Camagni, 1991; Asheim, 1996; Cooke, 2001).

Comprender cómo se desarrolla el fenómeno de la innovación y los factores que la determinan permitiría conocer también las sendas de desarrollo que ellos inducen (OCDE, 1997). En otras palabras, también es importante conocer los procesos de innovación para poder determinar la senda de desarrollo que de manera tácita o explicita es adoptada por el país (path dependency) o región. Y esto a su vez, es base fundamental para el diseño, implantación y evaluación de las políticas a desarrollar e implementar para poder, si es necesario, cambiar esa senda. En resumen, estudiar la innovación también es importante para la definición de estrategias de desarrollo del país o región en cuestión.

$\mathrm{Y}$ es en este sentido que la innovación es vista por los policy makers como una forma de obtener mejoras genuinas, sostenibles y acumulativas (RICYT/OEA/CYTED, 2001). Genuinas porque permiten generar condiciones competitivas a partir de la acumulación de conocimiento y el desarrollo de habilidades y el aprovechamiento de capacidades (naturales o adquiridas) haciendo que las firmas se destaquen sobre la competencia (Fajnzylber, 1998). Sostenible porque si bien se deben de utilizar los recursos naturales con los que cuenta el país o región, éstos deben de ser utilizados con el debido cuidado de evitar su degradación ni deteriorar el medio ambiente.. Y acumulativas porque determina el papel condicionante de la trayectoria futura (path dependency) que orienta la conducta tecnológica de las firmas y la generación de externalidades (spillovers) vinculadas a los procesos de aprendizaje que se presentan (Ocampo, 1991).

Pero además, dada las características de las firmas en América Latina, la innovación puede ayudar a eludir la competencia en precios y la dependencia del comercio exterior basada en los commodities que históricamente las han caracterizado. La innovación es referenciada como uno de los caminos para poder sostener un incremento sistemático de salarios sin afectar los niveles de competitividad. A su vez puede ser un elemento fundamental que permita evitar el deterioro de los términos de intercambio y los desequilibrios externos, permitiendo a su vez un mejor aprovechamiento de los recursos naturales (Lugones et al., 2007).

También es importante estudiar la conducta innovadora de las firmas debido a que muchos resultados del proceso de innovación sólo son parcialmente explicados por los inputs tradicionales de dicho proceso. Es así que, la literatura sobre innovación ha ido desplazando el eje del análisis desde la innovación propiamente dicha (actividades de I+D) hacia el esfuerzo tecnológico, las actividades de innovación a nivel de la firmas o las características y conductas de las firmas (Pavitt el al., 1987; Acs \& Audretsch, 1991; Cohen, 1995; Geroski, 1995; Patel \& Pavitt, 1995). 
Por último, se ha argumentado que la innovación es un proceso circular, complejo y con elementos interactivos que determinan la posibilidad de que se den procesos de aprendizaje colectivo entre los diferentes agentes que forman el Sistema Nacional de Innovación (S.N.I.). Un Sistema Nacional de Innovación (S.N.I.) se define como aquel sistema constituido por las organizaciones e instituciones de un país que influyen en el desarrollo, difusión y uso de las innovaciones (Edquist, 1997a y OCDE, 1999 citado en Navarro, 2001; Lundvall 2007). De acuerdo a Lundvall (1992) el termino institución es utilizado tanto en el sentido de normas, reglas y leyes que modelan los comportamientos, como en el sentido de estructuras formales como ser empresas, universidades, laboratorios, etc. (Navarro, 2001). En Uruguay los principales agentes del S.N.I., además de las firmas y de la agencia pública de promoción de la innovación (Agencia Nacional de Investigación e Innovación - ANII), son: las Universidades, los Centros Tecnológicos, las Institutos de Formación Técnica, los Laboratorios, las Unidades de Vinculación Tecnológica, las Entidades de Intermediación Financiera, los Proveedores, los Clientes, las Empresas relacionadas, los Consultores y expertos y la Casa matriz (ANII, 2009).

Este enfoque introduce la idea de que el comportamiento de las empresas respecto a las actividades de innovación, y por lo tanto el resultado de dicho proceso, se ve afectado por el entorno en que operan (Lundvall, 2007; Navarro, 2009).

Para el caso de Uruguay, al ser las pequeñas y medianas empresas (PYMES) una parte fundamental del tejido empresarial de la economía (Camacho et al., 2010, p. 97), comprender como éstas pueden innovar, cooperar y en lo posible aprender en forma conjunta se vuelve crítico para la correcta planificación y aplicación de políticas de incentivos. Esto es así porque, las empresas pequeñas y medianas tienen grandes dificultades para llevar adelante actividades de innovación. Las PYMES tienen serias dificultades para afrontar los retos competitivos derivados de la permanente competencia e innovación que caracteriza el entorno de negocios actual (Parrilli et al., 2010). Concretamente, de acuerdo a Parrilli et al. (2010), carecen de recursos humanos y financieros para dedicar a actividades de I+D, por lo que deben ser objeto de especial atención en las políticas de incentivo a la innovación.

\section{Objetivo de la investigación}

Existe en la literatura un apoyo generalizado a la idea de que la cooperación y las vinculaciones contribuyen al aumento en las actividades de innovación de las empresas, pero sin embargo poco se ha investigado sobre los mecanismos específicos a través de los cuáles se intercambia la información y cuál es la dinámica de la innovación que lleva a que sea exitosa en esos ambientes (Ibrahim \& Fallah, 2005).

La colaboración con instituciones externas para llevar adelante las actividades de innovación ha sido comprobada como muy positiva para las empresas, debido a que dichas instituciones ofrecen recursos, en particular conocimiento e información, que las empresas carecen. De acuerdo a Faria et al. (2010), las actividades de cooperación con otras empresas o instituciones son vistas como oportunidades para acceder a recursos tecnológicos complementarios, que pueden contribuir a un desarrollo más rápido de las innovaciones, permitir acceso a nuevos mercados, generar economías de escala y de alcance, distribuir costos y diversificar riesgos. También la consideran como un medio muy eficaz para la reorganización de la actividad industrial, teniendo en cuenta los complejos procesos de I+D (Faria et al., 2010). Esta realidad, vista como una necesidad de cooperar y de obtener innovaciones exitosas, se supone que es aún mayor en economías en desarrollo como la de Uruguay, en donde como ya se ha expresado la gran mayoría de las empresas son PYMES.

El objetivo principal de la presente investigación está orientado a comprender la forma en que las vinculaciones establecidas con los diferentes agentes del S.N.I. afectan las actividades de innovación realizadas por las empresas manufactureras industriales uruguayas.

De acuerdo a lo expuesto, la investigación se guiará por la siguiente pregunta:

¿Cómo afectan las vinculaciones realizadas con los diferentes agentes del S.N.I. a las actividades de innovación llevadas adelante por las empresas manufactureras industriales uruguayas?

En particular, interesa analizar el caso de la vinculación con un agente específico del S.N.I.: la Universidad. Las políticas de innovación han reconocido que la universidad y los centros tecnológicos y/o de investigación son agentes claves para el fomento de la innovación industrial (Veja-Jurado et al., 2009).

Para realizar la investigación se utilizaron los micro-datos de las encuestas de actividades de innovación realizadas a las firmas de la industria manufacturera en Uruguay y que abarcan el período 20012009. Dichas encuestas fueron realizadas por el gobierno uruguayo, a través del Instituto Nacional de Estadísticas (INE) y de la Agencia Nacional de Investigación e Innovación (ANII).

\section{Vinculación-Cooperación e Innovación}

A pesar de que el fenómeno de la colaboración tecnológica existe desde finales del siglo XIX, es el creciente número de acuerdos interempresariales y de sectores e instituciones involucradas, lo que ha despertado el interés como objeto de estudio reciente (Vega-Jurado et al., 2009).

Por ejemplo, para Bayona Sáez et al. (2002), dado que la innovación tiene un carácter circular e interactivo y debido a la creciente complejidad que prevalece en muchos de los sectores de la economía, las empresas están obligadas a establecer relaciones con otras instituciones y organizaciones con el fin de poder llevar adelante sus actividades de I+D.

En este sentido, el análisis de los flujos de conocimiento es esencial para comprender la cooperación en innovación. Existen estudios que detectan una relación positiva y significativa entre los flujos de información externos y la decisión de cooperar en I+D. Encuentran que las empresas que presentan externalidades positivas importantes en los insumos utilizados en su proceso de innovación, son más propensas 
a participar activamente en acuerdos de cooperación en I+D. Las firmas que son más eficaces en la apropiación de los resultados de sus procesos de innovación, son también más propensas a cooperar en I+D (Cassiman \& Veugelers, 2002 citado en Faria et al., 2010).

Por lo tanto, el manejo de las externalidades resultantes de la información (insumos entrantes) y la apropiación tienen efectos importantes: las empresas más capaces de capturar el conocimiento de fuentes externas y que están mejor preparadas para proteger sus propios conocimientos tienen una mayor probabilidad de cooperar en I+D o en actividades de innovación conjuntas (Cassiman \& Veugelers, 2002; Abramovsky et al., 2008 citados en Faria et al., 2010). Sin embargo, también de acuerdo a López (2008) también es posible que una empresa que está mejor preparada podría estar menos dispuesta a cooperar (López, 2008 citado en Faria et al., 2010).

Es así que, en años recientes ha venido tomando relevancia en la literatura sobre innovación el tema de la cooperación en actividades de $\mathrm{I}+\mathrm{D}$. La relación de cooperación que más atención ha recibido es la que realizan firmas e instituciones dedicadas a la investigación e innovación (universidades, centros de investigación, laboratorios) (Vega-Jurado et al., 2009).

Este enfoque, surge de la constatación de que la innovación, al ser una actividad cada vez más compleja, costosa y con altos riesgos (por ser imprevisible), se ha vuelto cada vez más una actividad inaccesible para muchas firmas. Es gracias a la colaboración por lo tanto, que muchas PYMES pueden realizar esa actividad (Sánchez-González et al., 2009). De esa manera las firmas que no pueden realizar grandes inversiones en $\mathrm{I}+\mathrm{D}$ pueden acceder a recursos e información que no están en condiciones de generar por su cuenta.

De acuerdo a Vega-Jurado et al. (2009), "la cooperación con agentes externos ha adquirido importancia como estrategia de innovación empresarial, siendo un mecanismo relevante a través del cual las empresas pueden acceder a los conocimientos y habilidades complementarias que poseen sus socios" (Vega-Jurado et al., 2009, p. 2). La cooperación de las empresas con universidades y centros de investigación ha sido unos de los objetivos prioritarios de las recientes políticas de innovación implementadas en muchos países de la OCDE (Vega-Jurado et al., 2009). El intercambio de conocimiento y los procesos de aprendizaje intensivos que caracterizan estas actividades de cooperación, tienden a combinar activos complementarios y a crear sinergias (Becker \& Dietz, 2004; Dachs et al., 2008 citados en Faria et al., 2010). Además, la decisión de las empresas de cooperar abre el abanico de las opciones tecnológicas disponibles, ya que los acuerdos de cooperación favorecen la acumulación de conocimiento haciendo más probable que se conviertan en innovaciones tecnológicas y organizativas reales (Mowery et al., 1998; Caloghirou et al., 2003 citados en Faria et al., 2010). Las empresas que cooperan o establecen alianzas están expuestas a flujos de conocimiento más densos que las que no cooperan (Gomes-Casseres et al., 2006 citado en Faria et al., 2010).

Desde el punto de vista económico, los estudios realizados focalizan los esfuerzos en responder cuáles son los factores que determinan el establecimiento de las redes y los acuerdos de cooperación y sus efectos sobre el desempeño innovador de las firmas (Vega-Jurado et al., 2009). Por ejemplo, para Hamel (1991) y Steensma (1996) (citado en Vega Jurado et al., 2009) principal razón para establecer relaciones de cooperación radica en "la posibilidad de adquirir e internalizar las habilidades y competencias de los socios para, a partir de ellas, crear nuevas competencias en la organización" (Vega-Jurado et al., 2009, p. 6).

Pero como cada uno de estos actores tiene motivos diferentes para cooperar, es fundamental elegir el candidato adecuado al momento de hacerlo. Es necesario balancear los aspectos positivos de cooperar (acceso a información, costos menores que el hacerlo en solitario e incluso posibilidad de realizar la innovación) con los aspectos negativos (potencial pérdida involuntaria de información crítica) (SánchezGonzález et al., 2009).

Una posible forma de aproximación para poder comprender como actúa la cooperación o colaboración entre las empresas y su efecto sobre las actividades de innovación y las innovaciones realizadas, es estudiar las vinculaciones entre los agentes de un sistema de innovación.

Sánchez-González \& Herrera (2010), expresan la necesidad de reconocer la importancia de los agentes externos como fuente de información para las actividades de innovación de las empresas y para el desarrollo de nuevos productos. Aducen que, la complejidad y dinamismo de entorno empresarial actual impone, a la hora de llevar adelante actividades de innovación, la obligación de complementar la base interna de conocimientos de la empresa con conocimientos provenientes del exterior mediante la cooperación con otras empresas o agentes.

Los resultados de su investigación corroboran la existencia de efectos muy diversos de la cooperación en los indicadores de innovación y que estos efectos dependen mucho del agente con quién se coopera. El modelo propuesto por los investigadores explica alrededor del $80 \%$ de la variación en el esfuerzo innovador en el sector productivo español. Encuentran que la cooperación de las firmas con clientes y proveedores produce un aumento significativo y positivo en la intensidad de las actividades de innovación en los sectores productivos (Sánchez-González \& Herrera, 2010)

El estudio muestra también que la cooperación con los clientes ha sido el único tipo de cooperación que ha producido un estímulo importante, tanto en las actividades de innovación al interior de la firma como en la adquisición de tecnología al exterior de la misma.

En lo que refiere a la cooperación con los competidores, encuentran que ésta aumenta la intensidad de la I+D. Los autores opinan que este resultado se da porque al compartir las empresas ciertos conocimientos con sus competidores, tienen que encontrar formas de mantener una posición de ventaja sobre ellos, y una forma de hacerlo es justamente aumentar sus esfuerzos en I+D (Sánchez-González \& Herrera, 2010).

Otro resultado que encuentran es que la cooperación con las universidades aumenta la adquisición de tecnología externa. En cambio la cooperación con los agentes públicos y agencias relacionadas a la $\mathrm{I}+\mathrm{D}$ 
la reduce. Según los autores esto estaría revelando que las empresas se comprometen con la cooperación por razones diferentes y que en algunos casos la cooperación es vista como un medio para la obtención de tecnología y no como un mecanismo de apoyo para sus procesos de innovación. Según estos investigadores esto podría estar indicando comportamientos oportunistas más que de sincera colaboración o cooperación. El estudio también muestra que la cooperación con centros tecnológicos públicos reduce significativamente el desarrollo de la tecnología al interior de la firma (Sánchez-González \& Herrera, 2010).

Por su parte, Vega-Jurado et al. (2009) concluyen que la cooperación de las empresas españolas con agentes científicos está incentivada por características asociadas al tamaño de la empresa y a la intensidad tecnológica del sector al que pertenece. Pero según estos autores, el resultado más interesante es que dicha cooperación parece estar más asociada o motivada por el acceso a recursos financieros (a través de la participación de programas públicos de apoyo a la innovación), que por la búsqueda de conocimientos o habilidades complementarias del socio.

Una gran parte de los estudios realizados han analizado el efecto de la cooperación tomando como caso de estudio la industria manufacturera de un país en particular (Becker \& Dietz, 2004; Miotti \& Sachwald, 2003; Amara \& Landry, 2005; Belderbos et al., 2004; VegaJurado et al., 2009), aunque existen estudios más amplios, para un conjunto de países u otros sectores como por ejemplo el sector de los servicios (Kaiser, 2002; Caloghirou et al., 2004).

\section{Análisis empírico}

Para realizar el análisis de los microdatos de las encuestas de innovación proporcionados por la Agencia Nacional de Investigación e Innovación (ANII) se decidió utilizar el Modelo Lineal General (Generalized Linear Model - GLM por su sigla en inglés). Esto es debido a que todas las regresiones planteadas tienen como variable dependiente variables que son discretas (dicotómicas).

Este tipo de metodología ha sido utilizada por ejemplo por Vega Jurado et al. (2009). Esto autores han aplicado la metodología GLM a la encuesta de innovación española a los efectos de estudiar la cooperación de las empresas con agentes científicos y determinar su incidencia sobre su desempeño innovador. En todos los casos han utilizando en sus regresiones variables discretas (cualitativas).

El Modelo GLM es una generalización de la regresión de Mínimos Cuadrados Ordinarios (MCO). El modelo GLM relaciona la distribución aleatoria de la variable dependiente (la función de distribución) con la parte no aleatoria, a través de una función llamada la función de enlace.

Fue formulado por Nelder \& Wedderburn (1972), quiénes unificaron varios modelos estadísticos (regresión lineal, logística y de Poisson) en un solo marco teórico. Al hacerlo, lograron desarrollar un algoritmo general para la estimación de máxima verosimilitud que funciona para todos los modelos estadísticos anteriores y que puede ser extendido a otros muchos modelos.
En concreto, para verificar las relaciones planteadas en el modelo teórico se va a utilizar un modelo GLM específico: el modelo Logit. Este modelo permite definir variables dependientes dicotómicas, que es la característica de las variables que se quieren utilizar.

Para calcular los modelos econométricos se utilizó el software R versión 2.15.1 (R Core Team, 2012).

\subsection{Hipótesis de Trabajo y formulación matemática}

Las Vinculaciones con los diferentes agentes del S.N.I. son fundamentales para llevar adelante las actividades de innovación. Pero no todas las vinculaciones establecidas tienen realmente un efecto positivo en dichas actividades.

La Hipótesis de trabajo que se tratará de contrastar en este documento expresa que:

"Las vinculaciones que las empresas tienen con los diferentes agentes del Sistema Nacional de Innovación (S.N.I.) influye positivamente en las actividades de innovación que realizan"

Se tratará de establecer cuáles son esas vinculaciones críticas, es decir, las vinculaciones que realmente tienen efecto en esas actividades de innovación. Es así que, para contrastar la Hipótesis se ha definido el siguiente modelo:

$$
B_{i}=\alpha+\sum \beta_{j} A_{j}+\sum \gamma_{k} H_{k}+\varepsilon_{i}
$$

Donde:

$B_{i}$ es el Tipo de Actividad de Innovación $\boldsymbol{i}$ es: 1. I+D Interna, 2. I+D Externa, 3. Adquisición de Bienes de Capital, 4. Adquisición de Hardware, 5. Adquisición de Software, 6. Transferencia Tecnología \& Consultorías, 7. Ingeniería \& Diseño Industrial, 8. Diseño Organizacional \& Gestión, 9. Capacitación.

$A_{j}$ son las variables de control $j: 1$. Porcentaje de capital extranjero que presenta la firma 2. Si la firma pertenece a un grupo económico, 3. Edad de la firma, 4. Tamaño de la firma, 5. Ingresos por ventas de la firma, 6. Porcentaje de exportaciones sobre el total de las ventas.

$H_{k}$ son las Vinculaciones con los diferentes agentes del S.N.I. $k$ : 1. Universidades, 2. Centros Tecnológicos, 3. Institutos de Formación Técnica, 4. Laboratorios, 5. Unidades de Vinculación Tecnológica, 6. Entidades Intermediación Financiera, 7. Proveedores, 8. Clientes, 9. Empresas Relacionadas, 10. Otras Empresas, 11. Consultores y expertos, 12. Programas y Agencias del Gobierno de CT, 13. Casa Matriz.

La variable dependiente es una variable discreta y en cada caso corresponde a si la empresa realizó o no un tipo de actividad de innovación concreto. 


\section{Ajuste de idoneidad}

Para contrastar el ajuste e idoneidad del modelo propuesto, se calculó el CAIC (Consistent AIC) para cada una de las ecuaciones de los tres modelos descriptos anteriormente. Para ello se utilizó el paquete ResourceSelection, desarrollado para R (Lele ,2009; Lele et al., 2012).

El razonamiento para saber si al modelo es idóneo o adecuado es el siguiente:

Dado un conjunto de posibles modelos para los datos, el modelo preferido es el que tiene el menor CAIC. El CAIC no sólo recompensa la bondad de ajuste, sino que también incluye una penalización que es una función creciente del número de parámetros estimados. El paquete calcula el CAIC de la siguiente forma: CAIC $=\alpha^{*} \mathrm{AIC}+(1-\alpha)^{*} \mathrm{BIC}$, con AIC $=$ Akaike Information Criterion, $\mathrm{BIC}=$ Bayesian Information Criterion y $\mathrm{a}=0,5$. Las fórmulas de los criterios son: $\mathrm{AIC}=2 \cdot \mathrm{k}-2 \cdot \ln (\mathrm{L})$ y $\mathrm{BIC}=-2 \cdot \ln (\mathrm{L})+\mathrm{k} \cdot \ln (\mathrm{n})$ con $\mathrm{k}$ = número de parámetros a estimar, $\mathrm{L}=$ el valor máximo de la función de probabilidad para el modelo estimado y $\mathrm{n}=$ número de observaciones de la muestra. Idealmente tanto el AIC como el BIC deben ser lo más pequeños posibles (Lele, 2009).
Los dos modelos que se han definido para comparar los respectivos CAIC son, el modelo original (con todas las variables) y el modelo que incluye solo las variables de control. Con ello se pretende comprobar que, al agregar las variables "vinculaciones", dichas variables realmente contribuyan a la explicación de la variable independiente. Esto sucede si el CAIC del modelo completo es menor que el CAIC del modelo que solo incluye las variables de control.

\subsection{Resultados del modelo econométrico y aceptación de hipótesis}

En este apartado se analizaran los resultados contrastándolos contra la Hipótesis de trabajo. Los resultados del modelo para los tres períodos considerados se presentan en el Cuadro 1. La aceptación o rechazo de la Hipótesis para cada una de las vinculaciones de las empresas con los diferentes agentes del S.N.I. y tipos de actividades de innovación llevadas adelante por las empresas se presentan en el Cuadro 2.

Se acepta la Hipótesis para los coeficientes de la ecuación GLM que sean positivos, estadísticamente significativos (significación al 5\%) y que además se cumpla que el CAIC del modelo completo sea menor que el CAIC del modelo que incluye solo las variables de control. 
Cuadro 1

\section{Resultados Modelo Econométrico}

Vinculación con el Agente del S.N.I. è Actividad de innovación

Período 2001-2003

Actividad de innovación
Vinculación con el Agente del S.N.I. Universidades

Centros Tecnológicos

Institutos de Formación Técnica

Laboratorios

Unidades de Vinculación Tecnológica

Entidades de Intermediación Financiera

Proveedores

Clientes

Empresas Relacionadas

Otras Empresas

Consultores y Expertos

Agencias o Prog. Gubernamentales de C\&T

Casa Matriz

CAIC

CAIC Modelo solo Variables de Control

I+D Interna $\quad I+D$ Externa

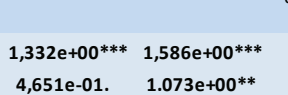

4,651e-01. $\quad 1.073 \mathrm{e}+00^{* *}$

2,81E-01 -6,46E-02

3,687e-01. 2,79E-01

$-5,13 \mathrm{E}-01 \quad-4,73 \mathrm{E}-01$

$-1,92 \mathrm{E}-01 \quad-1,55 \mathrm{E}-01$

2,98E-01 - -4,57E-01

5,146e-01* 5,63E-01

7,79E-02 2,10E-01

$-1,126 \mathrm{e}+00 * * \quad-9,02 \mathrm{E}-01$

$5,989 \mathrm{e}-01 * * \quad 1.299 \mathrm{e}+00 * * *$

2,07E-01 2,65E-02

$-4,18 \mathrm{E}-01 \quad-5,52 \mathrm{E}-02$

$\mathbf{7 8 0 , 2 0} \quad 366,39$

$836,47 \quad 390,47$

Fuente: Elaboración propia en base a los microdatos de la Encuesta de Innovación de la ANI Códigos de significatividad: (***)0,001 $\left.\left.\quad{ }^{* *}\right) 0,01 \quad{ }^{*}\right) 0,05 \quad$ (.) 0,1

\begin{tabular}{|c|c|c|c|c|c|c|}
\hline \multirow[b]{2}{*}{$\begin{array}{l}\text { Adquisición } \\
\text { de bienes de } \\
\text { capital }\end{array}$} & \multicolumn{3}{|c|}{ Actividad de innovación } & \multirow[b]{2}{*}{$\begin{array}{l}\text { Ingeniería y } \\
\text { diseño } \\
\text { industrial }\end{array}$} & \multirow[b]{2}{*}{$\begin{array}{c}\text { Diseño } \\
\text { organizacional } \\
\text { y gestión }\end{array}$} & \multirow[b]{2}{*}{ Capacitación } \\
\hline & $\begin{array}{l}\text { Adquisición } \\
\text { de hardware }\end{array}$ & $\begin{array}{l}\text { Adquisición de } \\
\text { software }\end{array}$ & $\begin{array}{c}\text { Transferencias } \\
\text { de tecnología y } \\
\text { consultoría }\end{array}$ & & & \\
\hline $4,05 E+02$ & $4,759 \mathrm{e}-01$ & $6,939 \mathrm{e}-01 * *$ & $6,697 \mathrm{e}-01^{*}$ & $6,874 \mathrm{e}-01 *$ & $4,18 \mathrm{E}-01$ & $1,067 e+00^{* * *}$ \\
\hline $2,24 E+02$ & $4,882 \mathrm{e}-01 *$ & 1,33E-01 & 4,310e-01 & $4,314 \mathrm{e}-01$ & $5,008 \mathrm{e}-01^{*}$ & $9,522 \mathrm{e}-01 * * *$ \\
\hline $2,94 E+02$ & $5,386 e-01$ & $4,08 \mathrm{E}-01$ & $4,35 \mathrm{E}-01$ & $-4,70 \mathrm{E}-01$ & $3,68 \mathrm{E}-01$ & $7,994 \mathrm{e}-01 * *$ \\
\hline 3,436e-01. & 2,12E-01 & $2,01 \mathrm{E}-01$ & $7,260 \mathrm{e}-01 *$ & $1,24 \mathrm{E}-01$ & 3,821e-01. & $3,25 \mathrm{E}-01$ \\
\hline$-8,10 E+02$ & $-1,540 \mathrm{e}+00 *$ & $-9,97 \mathrm{E}-01$ & $-1,04 \mathrm{E}-01$ & $-4,28 \mathrm{E}-01$ & $-9,04 \mathrm{E}-01$ & $-1,411 e+00 *$ \\
\hline $4,945 \mathrm{e}-01 *$ & $2,94 \mathrm{E}-01$ & $-3,18 \mathrm{E}-02$ & $-5,47 E-02$ & $-8,684 \mathrm{e}-01^{* *}$ & $1,06 \mathrm{E}-01$ & $-2,22 \mathrm{E}-01$ \\
\hline $6,612 \mathrm{e}-01 * *$ & $6,147 e-01 * *$ & $6,149 \mathrm{e}-01^{* *}$ & $-2,99 \mathrm{E}-01$ & $7,189 \mathrm{e}-01^{* *}$ & $1,82 \mathrm{E}-01$ & $4,883 e-01 *$ \\
\hline$-2,52 E+02$ & $2,22 \mathrm{E}-01$ & $9,55 \mathrm{E}-02$ & $2,43 E-02$ & 2,91E-01 & $4,960 \mathrm{e}-01^{*}$ & 2,90E-02 \\
\hline$-4,33 E+02$ & $-6,394 \mathrm{e}-01^{*}$ & $-5,307 e-01$ & $-6,64 \mathrm{E}-01$ & $-4,79 \mathrm{E}-01$ & $-2,21 \mathrm{E}-01$ & $-2,52 \mathrm{E}-01$ \\
\hline $5,554 \mathrm{e}-01$ & 1,93E-01 & $-4,37 \mathrm{E}-01$ & $-3,87 \mathrm{E}-01$ & $-7,16 \mathrm{E}-02$ & $-1,13 E-02$ & $-4,52 E-01$ \\
\hline $6,962 \mathrm{e}-01^{* * *}$ & $7,027 e-01^{* *}$ & $5,974 \mathrm{e}-01 * *$ & $1,622 \mathrm{e}+00 * * *$ & $6,424 \mathrm{e}-01^{* *}$ & $7,999 \mathrm{e}-01 * * *$ & $1,218 \mathrm{e}+00^{* * *}$ \\
\hline $1,36 \mathrm{E}+02$ & $-2,71 \mathrm{E}-01$ & $-2,96 \mathrm{E}-02$ & $8,45 \mathrm{E}-02$ & $8,650 e-01 *$ & $1,46 \mathrm{E}-01$ & $3,73 \mathrm{E}-02$ \\
\hline$-1,65 E+02$ & $4,21 \mathrm{E}-01$ & $6,42 \mathrm{E}-01$ & $-3,97 \mathrm{E}-01$ & 5,99E-01 & $-7,94 \mathrm{E}-01$ & $7,56 \mathrm{E}-02$ \\
\hline 890,67 & 794,96 & 806,53 & 470,52 & 707,93 & 742,99 & 779,27 \\
\hline 915,37 & 826,82 & 812,84 & 493,96 & 720,09 & 759,81 & 894,82 \\
\hline
\end{tabular}

\section{Período 2004-2006}

Actividad de innovación

Vinculación con el Agente del S.N.I.

Universidades

Centros Tecnológicos

Institutos de Formación Técnica

Laboratorios

Unidades de Vinculación Tecnológica

Entidades de Intermediación Financiera

Proveedores

Clientes

Empresas Relacionadas

Otras Empresas

Consultores y Expertos

Agencias o Prog. Gubernamentales de C\&T Casa Matriz

CAIC

CAIC Modelo solo Variables de Control

I+D Interna

I+D Externa

$1.228 \mathrm{e}+00 * * * \quad 9,604 \mathrm{e}-01 *$

$1,16 \mathrm{E}-01 \quad 8,658 \mathrm{e}-01 *$

5.344e-01. 1,48E-01

7.232e-01** $2,98 \mathrm{E}-01$

8,11E-01 - $-1,09 \mathrm{E}+00$

$-4,72 \mathrm{E}-01 \quad-1,090 \mathrm{e}+00 *$

8.088e-01** $2,76 \mathrm{E}-01$

4.861e-01. 4,74E-01

$3,44 \mathrm{E}-02 \quad 3,81 \mathrm{E}-01$

$1,94 \mathrm{E}-01 \quad 3,84 \mathrm{E}-01$

3,60E-01 1,214e+00**

$1.847 \mathrm{e}+00 * * * \quad 1,466 \mathrm{e}+00 * *$

$1,55 \mathrm{E}-01 \quad 5,35 \mathrm{E}-01$

624,51

$743,36 \quad 319,57$

341,29

Fuente: Elaboración propia en base a los microdatos de la Encuesta de Innovación de la AN

Códigos de significatividad: (***) 0,001 $\quad$ (**) $0,01^{(*)} 0,05 \quad$ (.) 0,1

Vinculación con el Agente del S.N.I.
Universidades
Centros Tecnológicos
Institutos de Formación Técnica
Laboratorios
Unidades de Vinculación Tecnológica
Entidades de Intermediación Financiera
Proveedores
Clientes
Otras Empresas
Consultores y Expertos
Agencias o Prog. Gubernamentales de C\&T
Casa Matriz

\section{I+D Interna I+D Externa}

$1,583 e+00 * * * \quad 2,2510+00 * *$

9,566e-01*** $1,92 \mathrm{E}-01$

5,782e-01. 2,42E-01

9,038e-01*** $-4,56 \mathrm{E}-01$

$-1,437 \mathrm{e}+00 * \quad 7,47 \mathrm{E}-02$

$-4,926 \mathrm{e}-01 . \quad-2,50 \mathrm{E}-01$

4,178e-01. $\quad-1,10 \mathrm{E}-01$

$6,788 \mathrm{e}-01 * * \quad 1,061 \mathrm{e}+00 *$

$2,40 \mathrm{E}-01 \quad-2,31 \mathrm{E}-01$

6,687e-01** $\quad 1,861 \mathrm{e}+00^{* * *}$

$3,86 \mathrm{E}-01 \quad 3,66 \mathrm{E}-02$

$1,099 \mathrm{e}+00 * \quad 1,05 \mathrm{E}+00$

\section{Período 2007-2009}

Actividad de innovación

Adquisición Adquisición Transferencias capital consultoría

6,969e-01* 4,828e-01. 4,07E-01

5,901e-01* 3,88E-01 6,915e-01*

5,612e-01* 7,317e-01** $\quad-8,74 \mathrm{E}-02$

$2,96 \mathrm{E}-02 \quad 1,35 \mathrm{E}-01 \quad 5,07 \mathrm{E}-01$

$-8,70 \mathrm{E}-01 \quad-1,186 \mathrm{e}+00 * \quad-5,99 \mathrm{E}-01$

6,636e-01** $-1,45 \mathrm{E}-01 \quad-4,13 \mathrm{E}-01$

$1,756 \mathrm{e}+00 * * * \quad 1,158 \mathrm{e}+00^{* * *} \quad 8,753 \mathrm{e}-01 * *$

$-4,891 \mathrm{e}-01 * \quad 1,16 \mathrm{E}-01 \quad-3,31 \mathrm{E}-01$

$3,78 \mathrm{E}-01 \quad 1,37 \mathrm{E}-01$

$7,580 \mathrm{e}-01 * * * \quad 9,368$

,

$2,65 \mathrm{E}-01$

$1,843 \mathrm{e}+00^{* * *}$

9,133e-01** 3,95E-01 8,370e-01*

$1,83 \mathrm{E}-01$

$5,25 \mathrm{E}-01$

$-3,85 \mathrm{E}-01$
Ingeniería y

industrial
$1,094 \mathrm{e}+00^{* * *}$

Diseño

organizacional

y gestión

8,628e-01**

2,37E-01

4,903e-01. 2, $2,89 \mathrm{E}-01$

$-7,26 \mathrm{E}-01 \quad 3,43 \mathrm{E}-02$

2,84E-01 4,31E-02

$1,094 \mathrm{e}+00 * * * \quad 7,499 \mathrm{e}-01 *$

$-8,93 \mathrm{E}-02 \quad-7,09 \mathrm{E}-02$

$-5,99 \mathrm{E}-02 \quad-1,24 \mathrm{E}-02$

$1,014 \mathrm{e}+00^{* * *} \quad 1,739 \mathrm{e}+00 * * *$

Diseño

organizacional

y gestión

5,703e-01. $\quad 1,388 \mathrm{e}+00 * *$

8,387e-01** 3,36E-01

6,138e-01. 2,048e+00**

4,98E-01 7,856e-01**

$-5,43 \mathrm{E}-02 \quad-1,23 \mathrm{E}-01$

2,47E-01 -8,87E-02

6,229e-01. 7,520e-01**

$1,82 \mathrm{E}-01 \quad 7,34 \mathrm{E}-02$

$-4,47 \mathrm{E}-01 \quad-3,03 \mathrm{E}-01$

1,01E-01 2,20E-01

8,061e-01* 5,939e-01**

$1,037 \mathrm{e}+00 * \quad 8,095 \mathrm{e}-01$.

4,73E-01 2,85E-01

486,43

758,04
939,77

$\begin{array}{rrr}\mathbf{6 4 6 , 2 8} & \mathbf{2 8 3 , 8 6} & \mathbf{9 3 2 , 1 6} \\ \mathbf{8 0 2 , 5 7} & 313,46 & 1.126,72\end{array}$

723,11
786,39

498,36

Fuente: Elaboración propia en base a los microdatos de la Encuesta de Innovación de la ANI

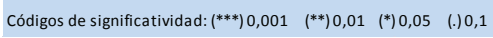

Fuente: elaboración propia

ISSN: 0718-2724. (http://jotmi.org)

Journal of Technology Management \& Innovation @ Universidad Alberto Hurtado, Facultad de Economía y Negocios. 
Cuadro 2

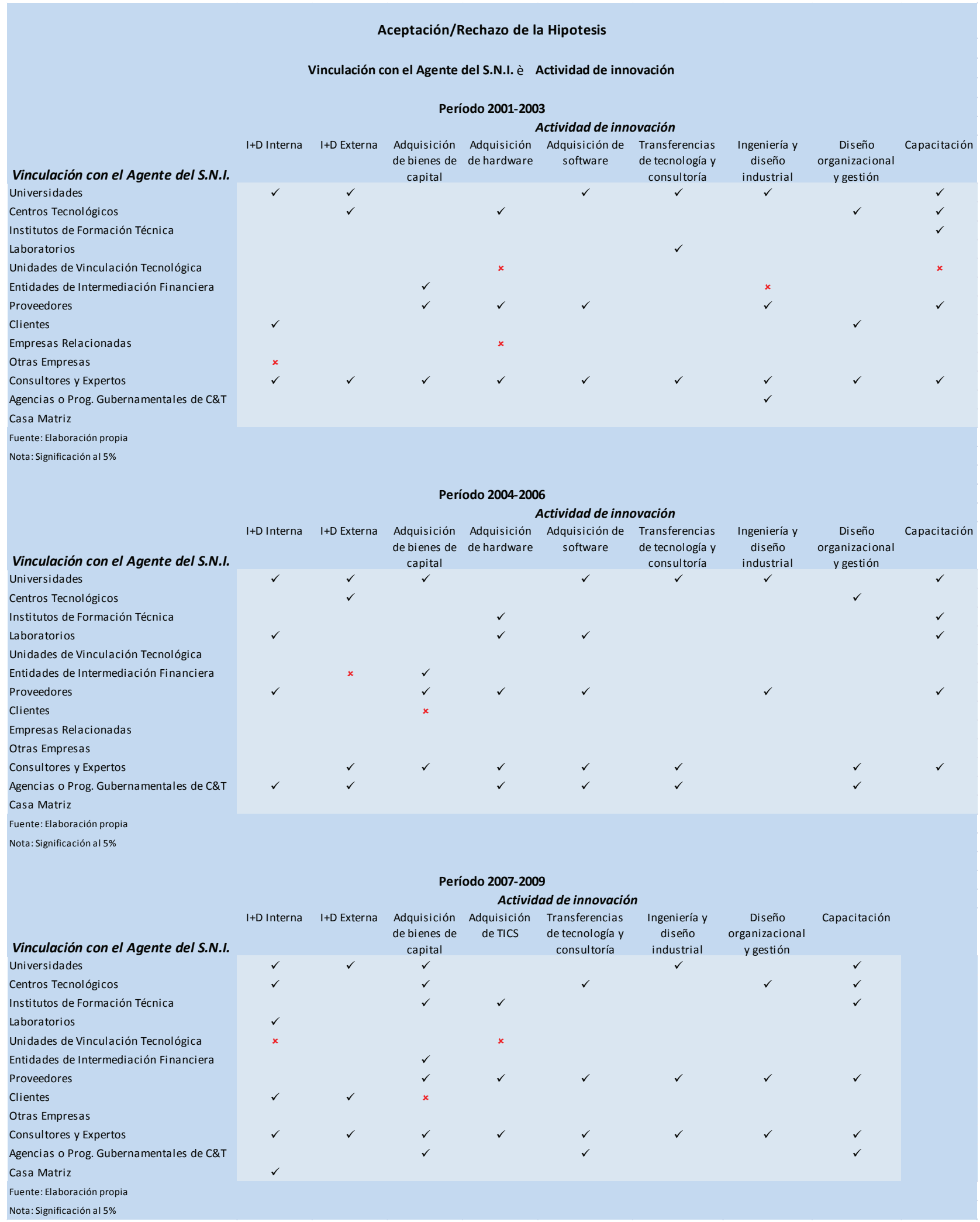

Fuente: elaboración propia

ISSN: 0718-2724. (http://jotmi.org)

Journal of Technology Management \& Innovation @ Universidad Alberto Hurtado, Facultad de Economía y Negocios. 
Como se puede observar en el Cuadro 2, para el período 2001-2003, se acepta la Hipótesis para las vinculaciones con los siguientes agentes del S.N.I.:

- Universidades para todas las actividades de innovación, excepto Adquisición de bienes de capital, Adquisición de hardware y Diseño organizacional y gestión.

- Centros Tecnológicos para las actividades I+D Externa, Adquisición de hardware, Diseño organizacional y gestión y Capacitación.

- Institutos de Formación Técnica para la actividad Capacitación.

- Laboratorios para la actividad Transferencias de tecnología y consultorías.

- Entidades de intermediación financiera para la actividad Adquisición de bienes de capital.

- Proveedores para las actividades Adquisición de bienes de capital, Adquisición de hardware, Adquisición de software, Ingeniería y diseño industrial y Capacitación.

- Clientes para la actividad Diseño organizacional y gestión.

- Consultores y expertos para todas las actividades de innovación.

- Agencias o Programas Gubernamentales de C\&T para la actividad de Ingeniería y diseño industrial.

En este período, para los agentes Unidades de Vinculación Tecnológica, Empresas relacionadas, Otras empresas y Casa matriz no se acepta la Hipótesis para ninguno de los tipos de actividad de innovación considerados.

Para el período 2004-2006 se acepta la Hipótesis para las vinculaciones con los siguientes agentes del S.N.I.:

- Universidades para todas las actividades de innovación, excepto Adquisición de hardware y Diseño organizacional y gestión.

- Centros Tecnológicos para las actividades I+D Externa y Diseño organizacional y gestión.

- Institutos de Formación Técnica para las actividades Adquisición de hardware y Capacitación.

- Laboratorios para las actividades I+D Interna, Adquisición de hardware, Adquisición de software y Capacitación.

- Entidades de intermediación financiera para la actividad Adquisición de bienes de capital.
- Proveedores para todas las actividades de innovación, excepto I+D Externa, Transferencias de tecnología y consultorías y Diseño organizacional y gestión.

- Consultores y expertos para todas las actividades de innovación, excepto I+D Interna e Ingeniería y diseño industrial.

- Agencias o Programas Gubernamentales de C\&T para todas las actividades de innovación, excepto Adquisición de bienes de capital, Ingeniería y diseño industrial y Capacitación.

En este período, para los agentes Unidades de Vinculación Tecnológica, Clientes, Empresas relacionadas, Otras empresas y Casa matriz no se acepta la Hipótesis para ninguno de los tipos de actividad de innovación considerados.

Para el período 2007-2009 se acepta la Hipótesis para las vinculaciones con los siguientes agentes del S.N.I.:

- Universidades para todas las actividades de innovación, excepto Adquisición de TICs, Transferencias de tecnología y consultorías y Diseño organizacional y gestión.

- Centros Tecnológicos para todas las actividades de innovación, excepto I+D Externa, Adquisición de TICs e Ingeniería y diseño industrial.

- Institutos de Formación Técnica para las actividades Adquisición de bienes de capital, Adquisición de TICs y Capacitación.

- Laboratorios para la actividad I+D Interna.

- Entidades de intermediación financiera para la actividad Adquisición de bienes de capital.

- Proveedores para todas las actividades de innovación, excepto I+D Interna, I+D Externa.

- Clientes para la actividad I+D Interna e I+D Externa.

- Consultores y expertos para todas las actividades de innovación.

- Agencias o Programas Gubernamentales de C\&T para todas las actividades Adquisición de bienes de capital, Transferencias de tecnología y consultorías y Capacitación.

- Casa matriz para la actividad I+D Interna.

En este período, para los agentes Unidades de Vinculación Tecnológica y Otras empresas no se acepta la Hipótesis para ninguno de los tipos de actividad de innovación considerados. 


\section{Análisis de resultados}

En el Cuadro 3 se presenta la evolución de los resultados de las vinculaciones del las empresas con los agentes del S.N.I. y las actividades de innovación llevadas adelante por las mismas, que están positivamente relacionadas y que son estadísticamente significativas ( la evolución de las Hipótesis aceptadas que se presentaron en el Cuadro 2):

\section{Cuadro 3}

\begin{tabular}{|c|c|c|c|}
\hline \multicolumn{4}{|c|}{ Evolución de la importancia de las Vinculaciones/Agente para las Actividades de Innovación } \\
\hline \multicolumn{4}{|c|}{ Vinculación con el Agente del S.N.I. è Actividad de innovación } \\
\hline \multirow[b]{2}{*}{ Vinculación con el Agente del S.N.I. } & \multicolumn{3}{|c|}{ Período } \\
\hline & 2001-2003 & 2004-2006 & 2007-2009 \\
\hline Universidades & $\begin{array}{l}\text { Todas las act. (excp. Adq. bs. cap., } \\
\text { Adq. hard., Diseño. org. y gest.) }\end{array}$ & $\begin{array}{c}\text { Todas las act. (excp. Adq. hard. y } \\
\text { Diseño. org y gest.) }\end{array}$ & $\begin{array}{c}\text { Todas las act. (excp. Adq. TICs, } \\
\text { Trans. tec. y consult., Diseño. } \\
\text { org. y gest.) }\end{array}$ \\
\hline Centros Tecnológicos & $\begin{array}{l}\text { I+D ext., Adq. hard., Diseño. org. y } \\
\text { gest., Capac. }\end{array}$ & I+D ext., Diseño. org. ygest. & $\begin{array}{l}\text { Todas las act. (excp. I+D Ext., } \\
\text { Adq. TICs, Ing. y diseño ind.) }\end{array}$ \\
\hline Institutos de Formación Técnica & Capac. & Adq. hard., Capac. & Adq. bs. cap., Adq. TICs, Capac. \\
\hline Laboratorios & Transf. tec. y consult. & $\begin{array}{l}\text { I+D Int., Adq. hard., Adq. soft., } \\
\text { Capac. }\end{array}$ & I+D Int. \\
\hline \multicolumn{4}{|l|}{ Unidades de Vinculación Tecnológica } \\
\hline Entidades de Intermediación Financiera & Adq. bs. cap. & Adq. bs. cap. & Adq. bs. cap. \\
\hline Proveedores & $\begin{array}{l}\text { Adq. bs. cap., Adq. hard., Adq. } \\
\text { soft., Ing. y diseño ind., Capac. }\end{array}$ & $\begin{array}{c}\text { Todas las act. (excp. I+D Ext., } \\
\text { Trans. tec. y consult., Diseño org. y } \\
\text { gest.) }\end{array}$ & $\begin{array}{c}\text { Todas las act. (excp. I+D Int. e } \\
\text { I+D Ext.) }\end{array}$ \\
\hline Clientes & I+D Int., Diseño. org. y gest. & & I+D Int., I+D Ext. \\
\hline \multicolumn{4}{|l|}{ Empresas relacionadas } \\
\hline \multicolumn{4}{|l|}{ Otras Empresas } \\
\hline Consultores y Expertos & Todas las act. & $\begin{array}{l}\text { Todas las act. (excep. I+D Int. e } \\
\text { Ing. y diseño ind.) }\end{array}$ & Todas las act. \\
\hline Agencias o Prog. Gubernamentales de C\&T & Ing. y diseño ind. & $\begin{array}{l}\text { Todas las act. (excep. Adq. bs. } \\
\text { cap., Ing. y diseño ind.) }\end{array}$ & $\begin{array}{l}\text { Adq. bs. cap., Trasnf. tec. y } \\
\text { consult., Capac. }\end{array}$ \\
\hline Casa Matriz & & & I+D Int. \\
\hline Fuente: Elaboración propia & & & \\
\hline
\end{tabular}

Fuente: elaboración propia

Se puede observar en el cuadro que las tres vinculaciones que tienen efectos positivos en una mayor cantidad de actividades de innovación y que se mantienen en los tres períodos son las realizadas con los agentes del S.N.I.: Universidades, Consultores y expertos y Proveedores.

En el caso de la vinculación con las Universidades, los efectos positivos se presentan también en casi todas las actividades de innovación. Se detecta un efecto positivo y estable en el tiempo sobre las actividades de I+D (Interna y Externa), Ingeniería y diseño industrial y Capacitación.

La vinculación con los Consultores y expertos ha sido constante en todo el período de estudio (2001-2009). Se han detectado efectos positivos para todas las actividades de innovación en los períodos 2001-2003 y 2007-2009. En el período 2004-2006 se deben excluir las actividades de I+D Interna e Ingeniería y diseño industrial).

La vinculación con los Proveedores ha ido creciendo en importancia. Eso se puede observar en la cantidad de actividades de innovación que afecta positivamente a través de los períodos de estudio. En el período 2001-2003 esta vinculación solo afectaba positivamente la realización de actividades relacionadas a la adquisición de bienes de capital o de TICs (software y hardware). Pero a partir del período 2004-2006, tiene un efecto positivo en la mayoría de las actividades de innovación llevadas adelante por las firmas. Para el último período presenta un efecto positivo para casi todas las actividades. La única actividad en la cual no presenta un efecto positivo es en la I+D Externa.
La vinculación con los Institutos de Formación Técnica ha evolucionado a través del tiempo. En el período 2001-2003 dicha vinculación solo presentaba efectos positivos sobre las actividades de Adquisición de hardware y Capacitación. A partir del período 20042006 se le agregan a las anteriores actividades, las actividades de I + D Interna, Adquisición de bienes de capital, y Diseño organizacional y gestión. En el período 2007-2009, las actividades sobre las cuales esta vinculación presenta efectos positivos son las mismas que las del período anterior con la excepción de que la actividad Ingeniería y diseño industrial ya no presenta coeficiente estadísticamente significativo.

En lo que se refiere a la vinculación con los Laboratorios se puede observar que ésta mantiene efectos positivos a través de los períodos de estudio sobre las actividades de I+D Interna y sobre la Adquisición de TICs y Capacitación.

En cambio, la vinculación con los Clientes si bien tiene efecto positivos sobre las actividades de innovación, solo se relaciona con las actividades de I+D (Interna y Externa) y en el período 2001-2003 con la de Diseño organizacional y gestión. Y se puede observar en el Cuadro 3 que, la vinculación con los Proveedores es mucho más prolífica, tanto en su alcance en cantidad de actividades de o como en su evolución a través del tiempo, que la que se puede detectar con los Clientes. 
A partir del período 2004-2006 se puede apreciar que la vinculación con el agente Agencias o Programas Gubernamentales de C\&T se vuelve importante para prácticamente todas las actividades de innovación. Seguramente el establecimiento de la ANII y sus programas tienen que ver con este efecto.

Es interesante el hecho de que se confirme mediante el test de mediación el papel esperado para las Entidades de Intermediación Financiera. Como se puede observar en el Cuadro 3, la vinculación de las empresas con ese agente del S.N.I. afecta positivamente la actividad Adquisición de bienes de capital, que como ya se expresó es la principal actividad de innovación llevada adelante en el período de estudio.

Por último, se debe destacar que para los agentes Unidades de Vinculación Tecnológica, Empresas relacionadas y Otras empresas no se han detectado la existencia de efectos positivos y estadísticamente significativos en ninguno de los tres períodos estudiados.

\section{Conclusiones}

De acuerdo a lo expuesto se puede concluir que:

1. Las Vinculaciones establecidas (y las posibles relaciones de cooperación), y que son críticas para las actividades de innovación llevadas adelante por las firmas, se han incrementado en cantidad a través de los períodos de estudio y para todas las actividades de innovación consideradas. Los principales agentes vinculados o contactados fueron: Universidades, Consultores y expertos, Proveedores, Institutos de Formación Técnica, Laboratorios, Clientes y la Agencias o Programas Gubernamentales de Ciencia \& Tecnología. Es importante destacar que, si bien los agentes vinculados o contactados por las firmas generalmente no cambian a los largo de los períodos de estudio, sí cambia para que actividades de innovación son contactados.

2. Si se fija el foco de atención en esto último, se pueden determinar "roles" específicos para cada uno de los agentes. Se podría decir que existen agentes que se los contacta para todo tipo de actividades y hay agentes que solo son contactados para actividades muy específicas. Se definirán a los primeros como agentes "generalistas" y a los segundos como agentes "especializados".

En el primer grupo se pueden incluir a los agentes del S.N.I.: Consultores y expertos (que son utilizados para todas las actividades de innovación), a las Universidades (casi todas las actividades de innovación) y a los Proveedores (en la mayoría de las actividades de innovación). En el segundo grupo a los Institutos de Formación Técnica, a los Laboratorios, a los Clientes y a la Agencias o Programas Gubernamentales de Ciencia \& Tecnología.
En el caso de la Universidad, el que se hayan detectado efectos positivos sobre casi todas las actividades de innovación es un hallazgo que se puede clasificar como muy propio de la Universidad uruguaya. Si bien es cierto que, generalmente los vínculos entre la Universidad y la industria o las firmas y sus respectivas redes son importantes y más frecuentes en las actividades de I+D. Esto se debe a que los conocimiento utilizados en este tipo de actividades de innovación son más a menudo codificados que de otros tipo y por lo tanto las innovaciones en consecuencia son impulsadas por la ciencia (science-driven) (Asheim et al., 2011).

Para los otros agentes se detectan roles más específicos y en algunos casos se podría decir que tradicionales. Los Institutos de Formación Técnica tienen efecto principalmente en la actividad de innovación Capacitación (aunque para los períodos 2004-2006 y 2007-2009 también se detecta que la vinculación de las firmas con este agente afecta positivamente las actividades de innovación Adquisición de TICs y de Bienes de capital). Los Laboratorios son utilizados para las actividades de I+D Interna, Adquisición de TICs y Capacitación. Los Clientes para las actividades de I+D tanto Interna como externa. Por último, la vinculación con la Agencia o Programas Gubernamentales de C\&T se enfoca principalmente en las actividades de Adquisición Bienes de Capital, Transferencias de Tecnología y Consultorías, Capacitación e I+D Interna.

3. Si bien de los resultados del estudio se puede afirmar que las vinculaciones de las empresas industriales manufactureras uruguayas con el agente Proveedores están bastante desarrolladas y parecen ser críticas, no parece ser así para el caso de los Clientes (al menos en su efecto directo sobre las Actividades de Innovación). Como se ha señalado la vinculación de las firmas con dichos agentes esencialmente afectan las actividades de innovación de I+D (interna y externa) pero no presentan ningún efecto sobre el resto de las actividades de innovación. La vinculación con los Proveedores es mucho más prolífica, tanto en su alcance en cantidad de actividades de innovación como en su evolución a través del tiempo, que la que se pudo detectar con los Clientes.

Este resultado está en línea con la investigación de Camacho et al. (2010). Estos autores señalan que las empresas uruguayas: “... en una de las preguntas que se puntuaron más bajo es en su habilidad de trabajar con inputs de otros orígenes. Lo que podría estar reflejando una fuerte vinculación entre la empresa y los proveedores" (Camacho et al., 2010, p. 98).

El resultado también es coherente con los tipos de actividad de innovación más importantes que se detecta que realizan las empresas uruguayas en el período de estudio: Ad- 
quisición de bienes de capital, Capacitación y Adquisición de TICs (Silveira, 2013). Las tres son actividades de innovación en donde la relación con el proveedor es fundamental, tanto en la etapa de diseño, como en la puesta en operación y en el seguimiento de los resultados de su implementación. Para el caso de la adquisición de bienes de capital, el proveedor es el que conoce en detalle los aspectos técnicos del bien adquirido y su asesoramiento en la adaptación a las necesidades del cliente así como la guía para el mantenimiento del bien, pueden ser razones para una relación de cooperación fluida. La capacitación generalmente la ofrece el propio proveedor teniendo en cuenta las necesidades del cliente. Por último, la adquisición de TICs, presentaría características similares a la de la adquisición de bienes de capital, aunque tal vez el tema del mantenimiento (upgrade) sea más importante,

Por último se debe aclarar que, si bien se ha podido comprobar en esta investigación que ciertos agentes (la Universidad entre los más importantes), tienen efectos positivos y significativos sobre las diversas actividades de innovación que llevan adelante las empresas industriales uruguayas en el período 2001-2009, queda pendiente para futuras investigaciones:

- Corroborar si dichos efectos siguen siendo válidos en el período 2010-2012 y extender la investigación al sector servicios. Para ello se analizarán los micro-datos de la última Encuesta de Innovación en la Industria y Servicios realizada por la ANII.

- Comprobar si ese efecto sigue siendo válido para el caso de las innovaciones realmente realizadas. Es claro que, si bien las actividades de innovación son una causa directa de innovaciones reales, no todas ellas llevan a innovaciones exitosas.

Esto puede significar que los efectos positivos de las vinculaciones de las empresas con los agentes considerados (entre ellos la Universidad) no necesariamente van a tener un efecto en las innovaciones exitosas. Esto último se puede considerar como una limitación importante de la presente investigación que debe ser subsanada en el futuro.

\section{Agradecimientos}

Los autores agradecen al Instituto de Competitividad de la Universidad Católica del Uruguay por el apoyo recibido y a la Agencia Nacional de Investigación e Innovación por haber proporcionado los micro-datos de las Encuestas de Innovación, insumo fundamental para la realización de esta investigación.

\section{Bibliografía}

Acs, Z. \& Audretsch, D. (1991). R\&D, Firm Size and Innovative Activity. Innovation and Technological Change: An International Comparison, Z. J.
Amara, N. \& Landry, R. (2005). Sources of innovation as determinants of novelty of innovation in manufacturing firms: evidence from the 1999 statistics Canada innovation survey. Technovation 25, 245-259.

ANII (2009). Informe de Resultados de la I Encuesta de Actividades de Innovación en Servicios. Montevideo.

Aranguren, M. J. \& Wilson, J (2010). Rethinking Territorial Competitiveness: What does it mean for a place to be "competitive"? Estudios Empresariales 134. Revista Cuatrimestral 2010/3. Universidad de Deusto.

Asheim, B. T. (1996). Industrial districts as "learning regions": a condition for prosperity. European Planning Studies 4 (4), 379-400.

Asheim, B. T., Moodysson, J. \& Todtling, F. (2011). Constructing Regional Advantage: Towards State-of-the-Art Regional Innovation System Policies in Europe? European Planning Studies 19 (7), 1133-1139.

Bayona Sáez, C., Gracía Marco, T. \& Huerta Arribas, E. (2002). Collaboration in R\&D with universities and research centers: an empirical study of Spanish firms. R\&D Management 32 (4), 321-341.

Becker, W, \& Dietz, J. (2004). R\&D cooperation and innovations activities of firms-evidence for the German industry. Research Policy 33, 209-223.

Belderbos, R., Carree, M. \& Lokshin, B. (2004). Cooperative R\&D and firm performance- Research Policy 32, 1477-1492.

Caloghirou, Y., Kastelli, I. \& Tsakanikas, A. (2004). Internal capabilities and external knowledge sources: complementes or substitutes for innovative performance? Technovation 24 (1), 29-39.

Camacho, M., Jung, A., Horta, R. \& García, S. (2010). ¿Cómo innovan las empresas exitosas en Uruguay?: una aplicación del modelo "la cometa de la innovación”. Instituto de Competitividad. Universidad Católica del Uruguay. Montevideo.

Camagni, R. (1991). Innovation Networks. Spatial Perspectives. Camagni ed. London and New York: Bellhaven Press.

Cohen, W. M. (1995). Empirical studies of innovative activities. Stoneman (ed.), Handbook of the Economics of Innovation and Technological Change, pp. 182-264. Oxford: Blackwell.

Cooke, P. (2001). Regional innovation systems, clusters, and the knowledge economy. Industrial and Corporate Change 10 (4), 945-974.

Faria, P., Lima, F. \& Santos, R. (2010). Cooperation in innovation activities: The importance of partners. Research Policy 39, 1082-1092.

Fajnzylber, F. (1988). Competitividad internacional, evolución y lecciones. Revista de la CEPAL 36. 
Geroski, P. (1995). Markets for technology: knowledge, innovation and appropriability. Stoneman (ed.), Handbook of the Economics of Innovation and Technological Change. (pp. 90-131). Oxford: Blackwell.

Hamel, G. (1991). Competition for competence and inter-partner learning within international strategic alliances. Strategic Management Journal 12, 63-103.

Ibrahim, S. \& Fallah, M. H. (2005). Drivers of Innovation and Influence of Technological Clusters. Engineering Management Journal 17 (3), 33-41.

Jaramillo, H., Lugones, G. \& Salazar, M. (2000). Normalización de Indicadores de Innovación Tecnológica en América Latina y el Caribe, Manual de Bogotá. OEA/ RICYT/ COLCIENCIAS/ CYTED/ OCT. Bogotá, Colombia.

Kaiser, U. (2002). An empirical test of models explaining research expenditures and research cooperation: evidence for the German service sector. Research Policy 20, 747-774.

Lele, S. R. (2009). A New Method for Estimation of Resource Selection Probability Function. Journal of Wildlife Management 73 (1), 122-127.

Lele, S. R., Keim, J. L. \& Solymos, P. (2012). ResourceSelection: Resource Selection (Probability) Functions for Use-Availability Data. $R$ package version 0.2-0.

http://CRAN.R-project.org/package=ResourceSelection

Lugones, G., Suarez, D. \& Gregorini, S. (2007), La Innovación como fórmula para mejoras competitivas compatibles con incrementos salariales. Evidencias en el caso argentino. Documento de Trabajo 36. Centro de Estudios sobre Ciencia, Desarrollo y Educación Superior. Argentina.

Lundvall, B. (1992). National Systems of Innovation: Towards a Theory of Innovation and Interactive Learning. London: Printer.

Lundvall, B. (2007). National Innovation Systems-Analytical Concept and Development Tool. Industry and Innovation 14 (1), 95-119.

Martínez-Sánchez, A., Vela-Jiménez, M. J., Pérez-Pérez, M. \& de LuisCarnicer, P. (2007). Flexibilidad Estratégica e innovación: el efecto moderador de las cooperación. Revista Europea de Dirección y Economía de Empresa 16 (4), 69-88.

Miotti, L. \& Sachwald, F. (2003). Co-operative R\&D: Why and with whom? An integrated framework of analysis. Research Policy 32, 1481-1499.

Navarro, M. (2001). Los sistemas nacionales de innovación: una revisión de la literatura. Documento de trabajo del Instituto de Análisis Industrial y Financiero. Universidad Complutense de Madrid, $\mathrm{N}^{\circ} 26$.

Navarro, M. (2009). Sistemas Nacionales de Innovación. Ekonomiaz 70, 24-59.
Nelder. J. A. \& Wedderburn. R. W. M. (1972). Generalized Linear Models. Journal of the Royal Statistical Society. Series A (General) 135 (3), 370-384.

Ocampo, J. A. (1991). Las nuevas teorías del comercio internacional y los países en vías de desarrollo, Pensamiento Iberoamericano 20, 193-214.

OCDE (1997). Proposed Guidelines for Collecting and interpreting Technological innovation Data, "Manual de Oslo". Eurostat.

Parrilli, M. D. Aranguren, M. J. \& Larrea, M. (2010). The role of Interactive Learning to Close the "Innovation Gap" in SME-Based Local Economics: A furniture Cluster in the Basque Country and its Policy Implications. European Planning Studies 18 (3), 351-370.

Patel, P. \& Pavitt, K. (1995). Patterns of technological activity: their measurement and interpretation. Stoneman (ed.), Handbook of the Economics of Innovation and Technological Change (pp. 14- 51). Oxford: Blackwell.

Pavitt, K., Robson, M. \& Townsend, J. (1987). The size distribution of innovating in the UK. Journal of Industrial Economics 35, 297-316.

Porter, M. (1990). The competitive Advantage of Nations. The Free Press, New York.

Porter, M. (2012). Microeconomics of Competitiveness. Core Concepts and Course Structure. Faculty Workshop, December 2012.

$R$ Core Team (2012). R: A language and environment for statistical computing. R Foundation for Statistical Computing, Vienna, Austria. ISBN 3-900051-07-0.

Retrieved from http://www.R-project.org/

RICYT/OEA/CYTED (2001). Manual de Bogotá.

Sánchez-González, G. \& Herrera, L. (2010). The influence of R\&D cooperation on innovatory effort. Innovation: Management, Policy and Practice 12 (3), 337-354.

Sanchéz-González, G., González-Alvárez, N. \& Nieto, M. (2009). Sticky information heterogeneous needs as determinig of R\&D cooperation with customers. Research Policy 38, 1590-1603.

Silveira, L (2013). Aprendizaje colectivo e innovación: el caso de las empresas manufactureras industriales uruguayas en el período 2001-2009. Tesis doctoral. Universidad de Deusto. España.

Simmie, J. (2006). Do clusters or innovation systems drive competitiveness? Cluster and Regional Development. Critical reflections and explorations. Asheim, Cooke \& Martin eds. Routledge London \& New York.

Vega-Jurado, J., Gutiérrez-Gracia, A. \& Fernández de Lucio, I. (2009). La Cooperación con Agentes Científicos y su Incidencia sobre el Desempeño Innovador de la Empresa. Ingenio CSIC-UPV. Working Paper 2009/05. 
Visser, E, J. \& Atzema, O. (2008). With or Without Clusters: Facilitating Innovation through a Differentiated and Combined Network Approach. European Planning Studies 16 (9), 1169-1188.
Wilson, J. (2010). Gobernanza y Desarrollo Socio-económico: Hacia nuevas políticas de competitividad. Separata del Boletín de Estudios Económicos Vol. LXV, No 200. Asociación de Licenciados en Ciencias Económicas por la Universidad Comercial de Deusto. Bilbao. 